\title{
On-line simulations of models for backward masking
}

\author{
GREGORY FRANCIS \\ Purdue University, West Lafayette, Indiana
}

\begin{abstract}
Five simulations of quantitative models of visual backward masking are available on the Internet at http://www.psych.purdue.edu/ gfrancis/Publications/BackwardMasking/. The simulations can be run in a Web browser that supports the Java programming language. This article describes the motivation for making the simulations available and gives a brief introduction as to how the simulations are used. The source code is available on the Web page, and this article describes how the code is organized.
\end{abstract}

Backward masking occurs when a briefly presented visual target stimulus becomes difficult to see because of the appearance of a mask stimulus that follows the target. Backward masking has been investigated in thousands of studies with a variety of experimental manipulations (see Breitmeyer \& Ogmen, 2000, and Enns \& Di Lollo, 2000, for recent reviews).

There are three reasons that interest in the properties of masking has been strong for decades. First, vision scientists use masking to explore interactions of the target and the mask signals and to identify key properties of the mechanisms involved in visual perception. Second, cognitive psychologists use backward masking as a means of interrupting the processing of target information. It is known that processing of a target does not stop with the physical disappearance of the target stimulus but that it can continue for at least a second after the stimulus has been turned off (Sperling, 1960). The presentation of a strong mask seems to halt further processing of the target stimulus as soon as the mask appears. Thus, by varying the timing between the offset of the target and the onset of the mask, the duration of processing can be controlled, and the details of cognitive mechanisms can be analyzed. Third, the properties of masking have been used to investigate aspects of various types of mental diseases (e.g., Braff \& Saccuzzo, 1981; Green, Nuechterlein, \& Mintz, 1994; Slaghuis \& Curran, 1999). Patients sometimes respond quite differently than normals under masking conditions.

Given the strong interest in masking and the frequency of its use as a tool for investigating perceptual, cognitive, and behavioral systems, it is perhaps surprising to note that there is currently no generally agreed upon theory of the mechanisms that are involved in producing masking effects. There is no shortage of theories, but none is gener-

This material is based on work supported by the National Science Foundation under Grant No. 0108905. Correspondence concerning this article should be addressed to G. Francis, Department of Psychological Sciences, Purdue University, 703 Third Street, West Lafayette, IN 47907-2004(e-mail: gfrancis@psych.purdue.edu). ally believed to properly account for the key data in the field. Researchers who have used masking as a tool to explore other issues generally have had an implicit theory that the mask interrupts processing or interferes with detection of the target properties. However, these ideas generally have not been rigorously investigated (usually because the researcher was actually interested in something other than masking per se). Even within the field of masking, theories often have only been described verbally, and sometimes without a description of the underlying mechanisms that would need to exist to instantiate a theory.

The field of masking would be enhanced if researchers understood and developed quantitative models of masking. A quantitative model is precisely defined, and its properties can be demonstrated conclusively. This specificity allows a model to make predictions and allows those predictions to be tested experimentally. These tests can, in turn, be used to further the development of the model and so initiate an upward spiral toward the creation of models that account for large amounts of data. In addition, quantitative models can be analyzed mathematically to further our understanding of the basic properties of the models. The latter analysis can help researchers who use masking as a tool to better understand how masking contributes to the primary effect they are studying.

There are several quantitative models of backward masking. In the 1970s, two neural network models were investigated and were shown to account for several properties of backward masking. Weisstein $(1968,1972)$ investigated a dual-channel neural network, whereas Bridgeman $(1971,1978)$ studied properties of a neural network with recurrent lateral inhibition. Anbar and Anbar (1982) elaborated a model of brightness perception to show that it accounted for some properties of backward masking. Francis (1997) showed that a neural model of cortical visual processing accounted for many properties of backward masking. Purushothaman, Ogmen, and Bedell (2000) showed that a recurrent neural network could produce oscillations that accounted for new data in backward masking. Finally, Di Lollo, Enns, and Rensink (2000) showed 
that a system with reentrant processing could account for their newly discovered object substitution masking effects. A recent analysis by Francis (2000) showed that, despite their differences, many of these models work according to the same underlying principles.

Although none of these models account for all of the data on backward masking, many of them use fairly simple principles to account for much of the experimental data. However, the contribution of the quantitative models appears not to have been recognized by many of the researchers that have studied or used backward masking. For example, Di Lollo et al. (2000) reported a new masking effect that they called object substitution. Di Lollo et al. suggested that a key property of this new masking effect ruled out existing theories of masking. The key effect was that strong masking could occur for a spatially impoverished mask (four dots around a target) when the target and the mask had common onsets but the mask stayed on after the target disappeared. The new effect is quite interesting and is a new experimental finding; however, Francis and Hermens (2002) showed, through computer simulation, that some of the quantitative models of masking can account for the effect without a change in parameters. Thus, even mathematically sophisticated researchers (Di Lollo et al. [2000] have proposed their own quantitative model) may not recognize the properties of existing quantitative models of backward masking.

The problem is even more severe in fields in which masking is used as a tool for studying other processes. Experimental studies in which masking has been used to investigate some aspect of cognition rarely have made reference to any of the models of backward masking. Likewise, clinical psychologists who have studied the effects of masking in mental disorders have never used the quantitative models to explain their findings.

This lack of reference to models is perhaps not surprising, because the quantitative models are difficult to un- derstand for individuals without a solid background in mathematics. Moreover, in many cases, the researcher would need to build a simulation of a model in order to use it to interpret experimental data. Building a simulation is a nontrivial task that can be a source of frustration even for researchers who have received formal training in model simulation. When someone is using masking as an experimental tool to investigate something else, it is perhaps unreasonable to expect them to assign resources toward development of a computer simulation of quantitative models of backward masking.

In a recent study by Francis (2000), computer simulations of several quantitative models of backward masking were developed. Most of these simulations were written in the Java programming language, so they can run on a variety of computers and can be easily distributed in Web browsers on the Internet. In an effort to promote understanding of the quantitative models of backward masking, I have created a graphical user interface that allows anyone to use these models. The model simulations are available on the Web at http://www.psych.purdue.edu/ gfrancis/ Publications/BackwardMasking/. Use of the models requires a Java-enabled Web browser (this includes most Web browsers) and access to the Internet. Full source code for the programs is also available as a download from the Web page.

The desired effect of making the model simulations widely available is twofold. First, it is hoped that researchers who want to learn more about quantitative models will use the simulations to help them better understand the properties of the models. There is sometimes a bootstrapping problem with model comprehension, where it is difficult to understand a model without a simulation but it is nearly impossible to build a simulation without understanding the model. Having an example of a model simulation available might make it easier to understand the properties of the model. Second, it is hoped that having the simulations

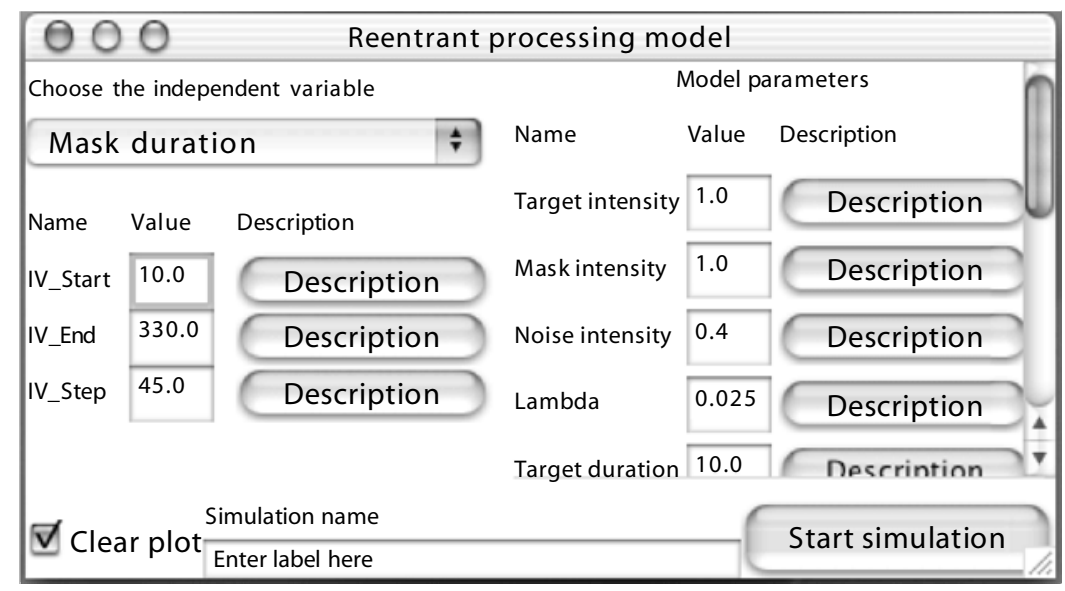

Figure 1. The graphical user interface for interacting with the reentrant processing model. The user interface is the same for every model. 
readily available will pique the interest of experimentalists to see whether the models can account for properties of their data. Such interest may motivate an experimentalist to further explore the characteristics of the models and may lead to more interpretations of experimental results with quantitative modeling.

\section{Models Available}

The models available on the Web site include only those models that do not require excessive amounts of computation. In general, a few minutes was considered an upper limit on how long people would wait before getting results from a simulation run. Models that meet this requirement include those discussed in Weisstein (1972), Bridgeman (1978), Anbar and Anbar (1982), Francis (2000), and Di Lollo et al. (2000). Not included are models by Francis (1997) and Purushothaman et al. (2000), because

\section{(A)}

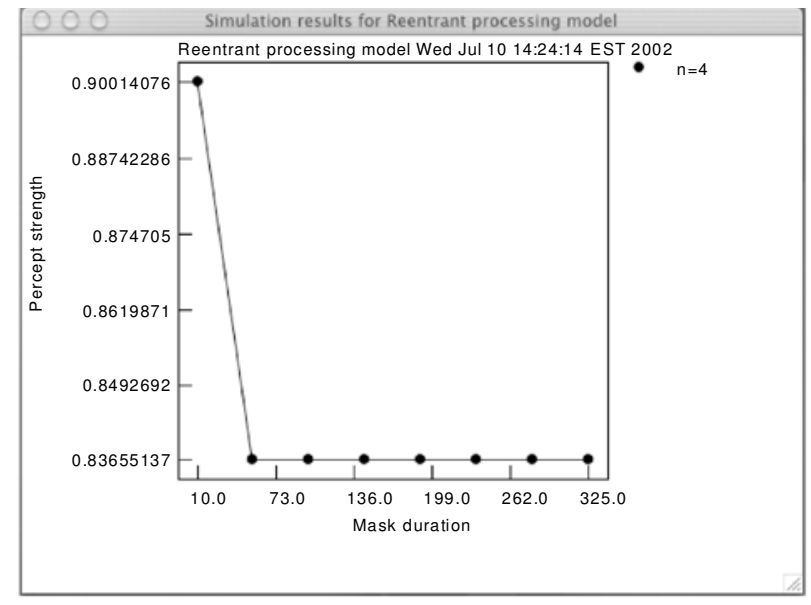

(C)

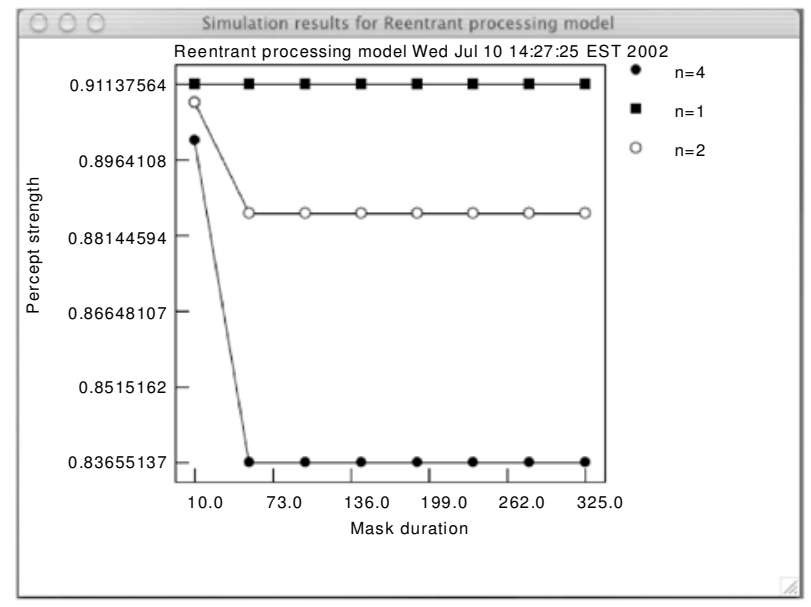

these models often must run for hours or days to generate results of interest.

A Java applet resides on the Web page to display buttons with names for the different models. Clicking on one of the buttons will cause a new window to appear with a graphical user interface (GUI) that allows the user to modify stimulus parameters and model parameters. For example, Figure 1 shows the window that appears after clicking on the Reentrant processing button. (All screen shots were created while running the simulations on a computer running Mac OS X, but the programs also work on MS Windows and Unix systems with Web browsers that support Java.)

Each simulation has the same type of GUI. The window is given a title that indicates which model is being simulated. The left side of the window contains information about an independent variable. This is the variable that is
(B)

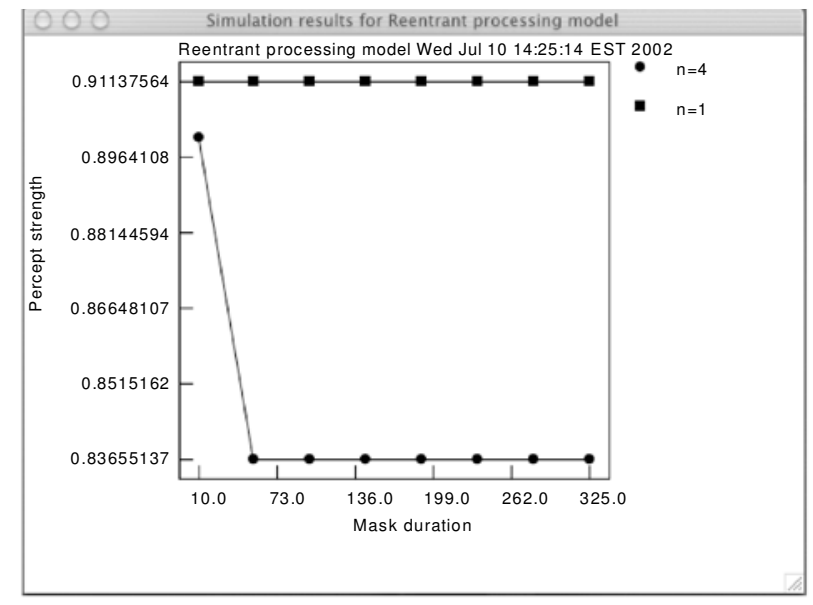

(D)

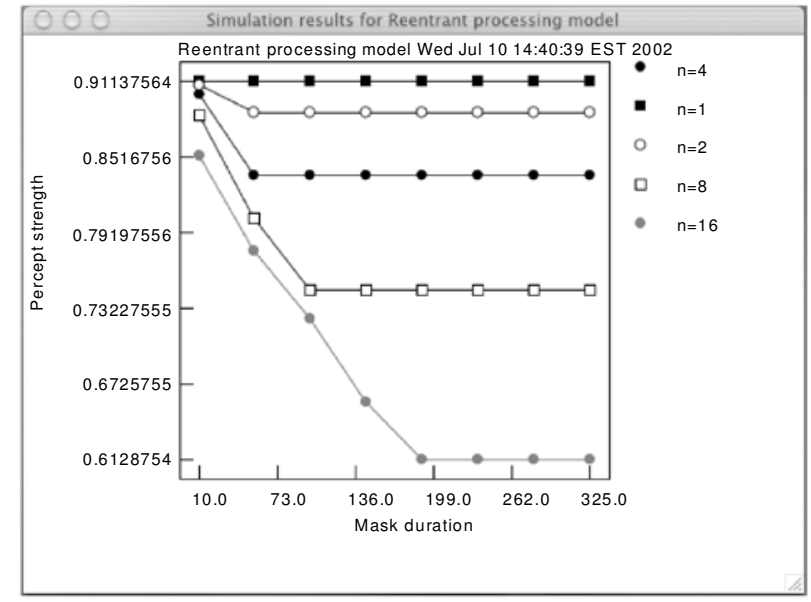

Figure 2. Simulation results for the reentrant processing model. After each run of the simulation, a data plot is produced. Panels A-D show the build up of plots as new data are added to previous simulation results. The final plot (panel D) is a replication of simulation results reported by Di Lollo, Enns, and Rensink (2000). 
systematically varied during a simulation run. After every run of a simulation, a graph is shown that plots the model's measure of the target percept strength as a function of the individual variable. Target percept strength is a model measure that corresponds to the visibility of the target. In different experiments, it would correspond to target brightness, percentage of correct detections, percentage of correct discriminations, and so on. The right side of the window lists each model parameter and stimulus parameter that can be set by the user. The bottom of the window lets the user set details of the simulation run. The checkbox labeled Clear plot determines whether the next simulation run should be displayed with previous simulation runs or whether it should generate an entirely new data plot. The text field labeled Simulation name allows the user to enter a name for the next simulation run. This name appears as a label in the legend of the data plot. Finally, clicking on the Start simulation button starts the simulation for the given set of parameters. As the simulation proceeds, this button's label is updated to roughly indicate the simulation's progress.

For every parameter, there is a button labeled Description. When this button is pressed, a window appears that provides a textual description of what the parameter corresponds to in the model and/or simulation. This information is provided to make it easier for the user to link the parameters in the simulation with the details of the models given in their original publications.
The worth of the simulations can be described by example. The first example will focus on the reentrant processing model of Di Lollo et al. (2000), because it is a new model and there is currently substantial interest in the issues raised by Di Lollo et al. A key property of the model of Di Lollo et al. concerns the effect of varying the number of distractor items when the target is presented. In the model, this is coded by the parameter $n$. Figure 2A shows the data plot that appears after one gives the simulation run the name $n=4$ and clicks on the Start simulation button. Additional simulation results are added to the graph by changing the parameter $n$, changing the simulation name, and unchecking the Clear plot checkbox. Figures 2B-2D show the model output as simulations for other values of $n$ are added to the graph.

Figure 2D is a replication of the results reported by Di Lollo et al. (2000) in their Figure 15A. It shows the effect of varying mask duration for different numbers of distractors in the target field. The main result is that there is an interaction of set size and mask duration. The curves in Figure 2 look slightly different from the curves in Figure 15A of Di Lollo et al., but this is because the values of mask durations are slightly different in the two sets of simulations.

Because the graph generated by the program is not of publication quality, the program also provides the simulated data in a plain text format. A menu option called Show data is available on each plot window. Selecting the Show data points menu item displays a new window that lists

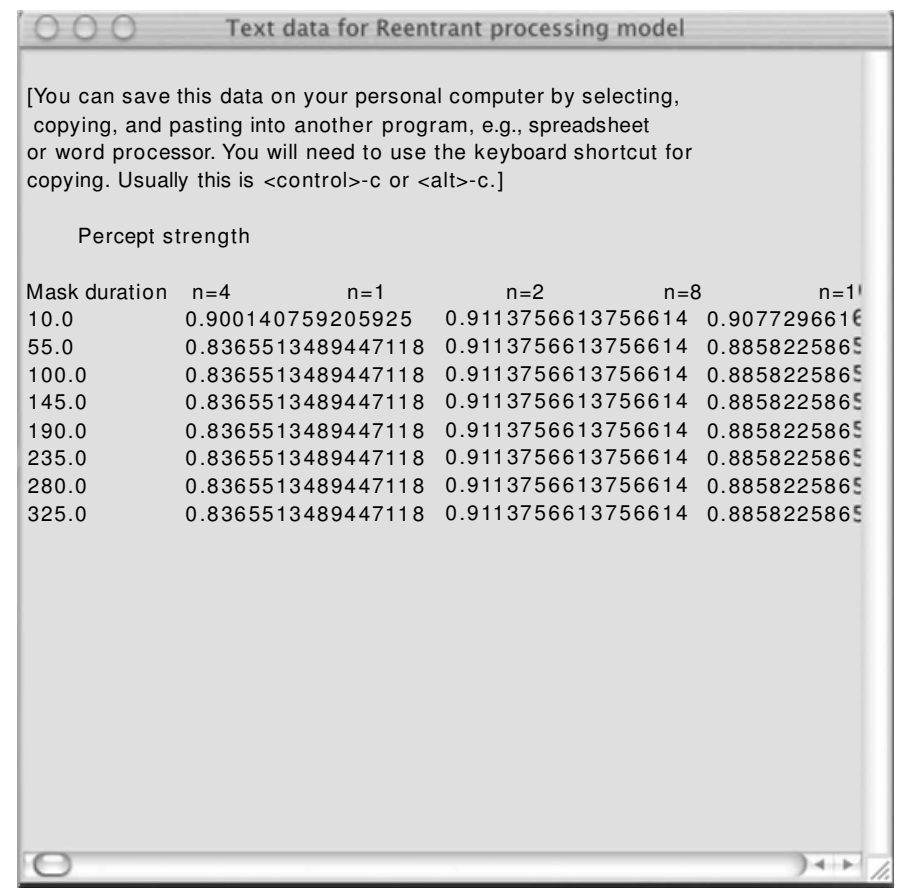

Figure 3. To produce better plots or to further analyze the simulated data, selecting the menu option Show data from each plot opens a window with a textual listing of the data points. The data can be selected, copied, and pasted into other programs. 
(A)

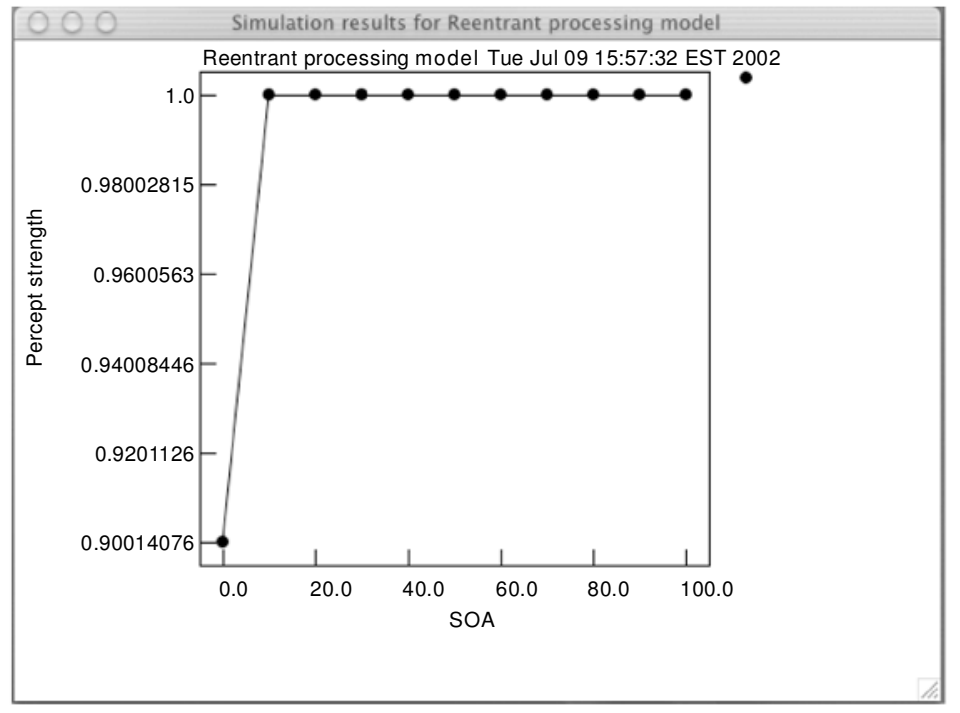

(B)

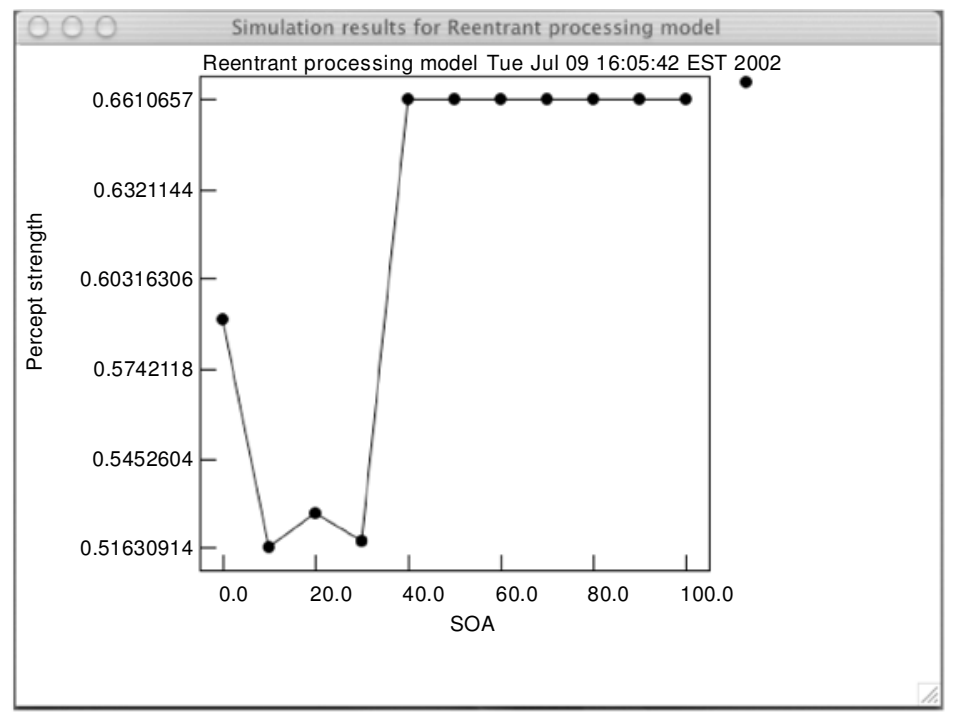

Figure 4. Simulation results when the reentrant processing model is tested under new conditions, varying the stimulus onset asynchrony (SOA) between the target and the mask stimuli. The results with the original model parameters are shown in panel $A$, and the results with a different set of parameters are shown in panel $\mathrm{B}$. The model can produce $\mathrm{U}$-shaped masking functions under some conditions.

the data in a textual format, as in Figure 3. These data can be selected, copied, and pasted into other programs (e.g., word processor, spreadsheet, text editor). Because columns are tab delimited, pasting the selected data into a spreadsheet should cause each number to be placed in its own cell. The data can thus be imported into the user's favorite plotting program to create higher quality graphs. The data can also be imported into a statistical analysis program for comparison with experimental data.
The default parameters when a simulation is started always correspond to the parameters used in the original description of the model. However, a user can change these parameters to explore the abilities of the model in other situations. For example, although the reentrant model of Di Lollo et al. (2000) was originally designed to account for effects of mask duration with common onset masking and variability in the number of distractors with the target, a researcher might be interested in seeing how it responds 
to variations in the stimulus onset asynchrony (SOA) between the target and the mask. A common experimental finding is that if the mask stimulus is weak relative to the target stimulus, the mask has its biggest effect at an intermediate SOA value. When target percept strength is plotted against SOA, the curve takes a U-shape. Accounting for the U-shaped masking function is considered a primary issue in the field of backward masking (Breitmeyer \& Ogmen, 2000; Francis, 2000).

Figure 4A shows the behavior of the model when the independent variable is SOA instead of mask duration. All the other parameters were the default values when the simulation started. The model predicts modest masking for common onset of the target and the mask and no masking for other SOAs. This finding does not provide evidence against the model of Di Lollo et al. (2000), because it was designed to explain other datasets. Interestingly, with a change of parameters, the model does seem to be able to produce U-shaped masking functions. By setting the target intensity to be 2.0 and $\lambda=0.95$, the model produces the curve in Figure 4B, which shows that the strongest masking occurs at intermediate SOA values.

This simulation finding is interesting because it suggests that Di Lollo et al.'s (2000) model, which was designed to account for common onset masking, might also be able to explain effects when the mask onset comes after the target offset. Of course, further research is needed to explore this possibility, and a simulation model is now available to support that research.
Similar investigationscan be made with the other model simulations. For example, Bridgeman's (1978) model uses recurrent lateral inhibition across a set of cells. The model includes longer time delays for inhibitory signals that must travel longer pixel distances. A researcher might wonder whether the time delays are necessary to produce the model's main effect (the existence of a U-shaped masking function). This can be explored in the model by setting the weights for distances greater than 1 pixel equal to zero. With zero signal being sent for the longer distances, such a system will have no time delays. Figure 5 shows the masking functions produced for the default parameters and for the simulation without time delays. Both produce a U-shaped masking function, which indicates that the time delays are not critical to producing the U-shaped masking function.

As a final example, Francis (2000) identified a novel method, called efficientmasking, for producing a U-shaped masking function. The default parameter values are those used in Francis (2003) to relate the model to experimental data. Figure 6A shows the kind of masking function produced by the model.

A researcher might wonder whether the efficient masking model could also account for the properties of common onset masking studied by Di Lollo et al. (2000). The answer is yes if one is willing to hypothesize that increases in set size lead to distributed attention, which makes the mask signal have a larger impact on the target signal (see Francis \& Hermens, 2002). Figure 6B shows simulation results that demonstrate this property.

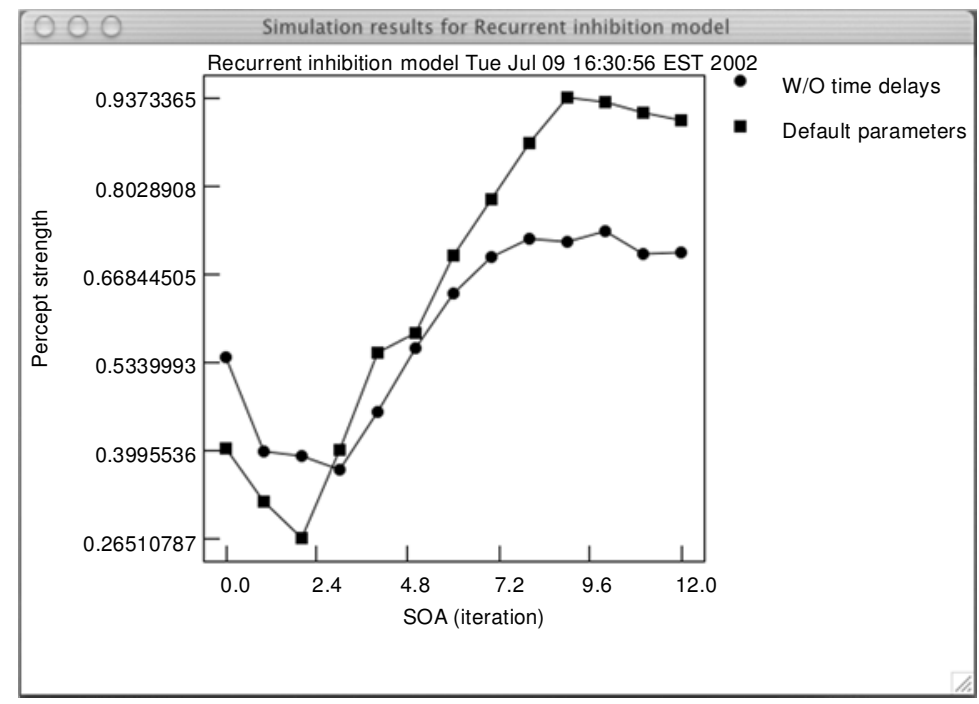

Figure 5. Simulation results for the recurrent inhibition model. The two curves differ depending on the weight given to spatial interactions beyond a cell's nearest neighbors. The curve with filled circles marking points is from a simulation with the weights set equal to zero. Setting these weights to zero also has the effect of removing time-delayed inhibition in the model. As the simulations show, $U$-shaped masking occurs regardless of this property of the model. 
(A)

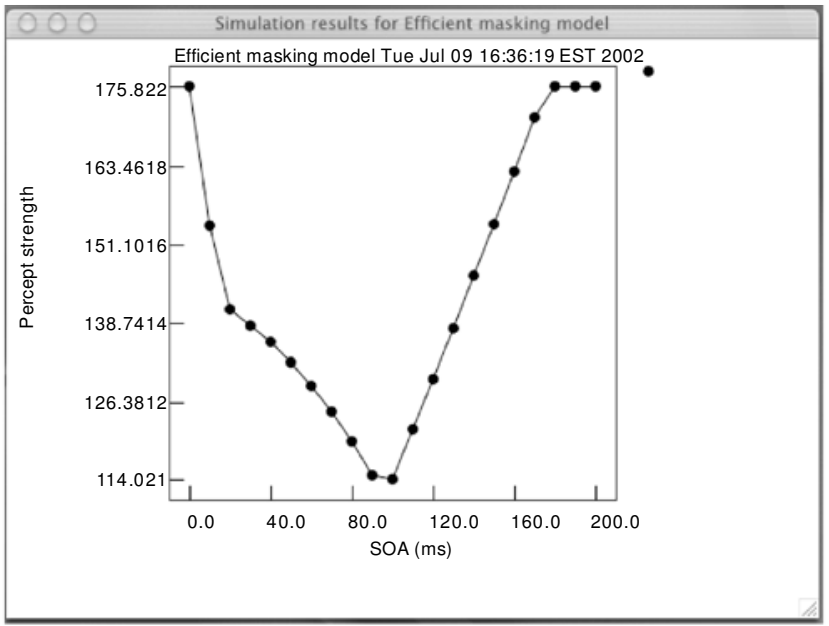

(B)

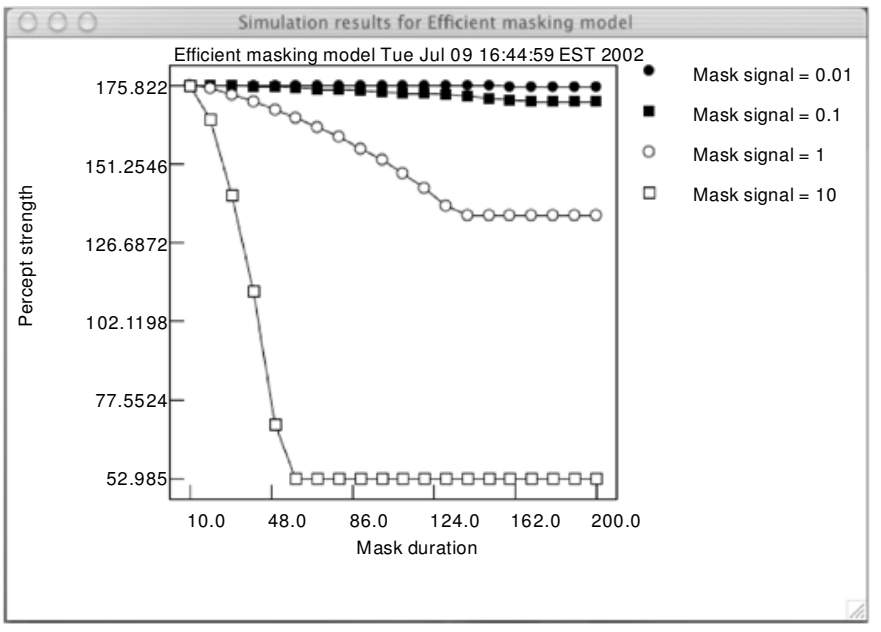

Figure 6. Simulation results for the efficient masking model. Panel A shows a U-shaped masking function produced by the default set of parameters. Panel B shows that the efficient masking model can match the basic properties of object substitution masking (see Figure 2D for comparison).

\section{Source Code}

Although one can use the simulation GUI to vary all of the model parameters and to vary the stimulus, there are some situations in which modification of the source code will be required. For example, one of the parameters in the model of Di Lollo et al. (2000) is interpreted as reflecting the rate at which items are searched in a visual display. For some of their simulations, this rate was changed within a run of the simulation to indicate precue and postcue rates. The simulations reported here cannot duplicate this effect, because the search rate can only be one value during a run of the simulation. However, it should be relatively easy for a programmer to modify the source code and allow for this possibility. More generally, a researcher may want to modify one of the models, which will require changes to the programming of the simulation. Thus, a discussion of how the source code is designed may be worthwhile.
The simulation programs are written in the Java programming language. They can be run as stand-alone programs or, as has been discussed so far, in a Web browser over the Internet. Java is an object-oriented language, with each object defined as a class that contains data and methods that manipulate the data.

Many of the classes define fairly general objects; for example, CloseableFrame.java defines a class that provides a window that closes upon request. Other classes do fairly complex operations. For example, SimPlotImage.java takes results from a simulation, draws an image of a data plot, and displays the image in a window.

The classes of interest for researchers who want to elaborate or modify the simulations are usually named after the researcher(s) who created the models (the one exception is for the efficient masking model, which is not named after Francis [2000] in order to avoid confusion with a dif- 
ferent model described in Francis [1997]). Thus, the classes are AnbarAnbar.java, Bridgeman.java, DiLolloEtAl.java, EfficientMasking.java, and Weisstein.java. Each of these classes defines the variables, parameters, and equations that make up the simulation of the model.

Every simulation program has a common design. The Java programming language has a convenient method of organizing similar types of programs, using abstract classes. An abstract simulation class was defined and called Simulation.java. The abstract class defines variables and methods that must be part of every simulation. The classes that define a particular model extend the abstract class and immediately acquire the data variables defined by the abstract class. Each particular model class must also provide a definition for each method defined in the abstract class. This ensures that each particular model has the basic properties of the abstract class.

The advantage to this arrangement is apparent in the design of the GUI (SimulationGUI.java). The type of model being simulated need not be specified for the GUI. Since every model is a version of the simulation class, certain variables always exist, and certain methods can always be called. Thus, if a new model simulation is created and is defined as an extension of the abstract simulation class, the GUI will automatically work with the new model simulation.

Thus, to make a new model simulation, it is necessary only to define a class along the lines of one of the existing models. This involves identifying the parameters for the model and identifying the model calculations. Of course, knowledge of the Java programming language is necessary to make these types of changes.

\section{Conclusions}

A set of programs that simulates five models of backward masking is available on the Internet. Either the programs can be run through a Web browser, or the source code can be downloaded, compiled, and run on a local computer. The programs provide a common interface for interacting with each simulation.

The model simulations will assist researchers interested in backward masking to explore the properties of these models and to thereby gain a better understanding of the types of interactions that may be involved in masking. The goal is to allow the properties of the quantitative models of backward masking to be understood and used by more researchers in the field of masking, by experimentalists who use masking as a tool to study other aspects of cog- nition, and by psychiatrists who relate masking effects to various mental conditions. If the need for these researchers to create their own simulations of the models is removed, perhaps the researchers will be motivated to use quantitative models to generate novel interpretations of experimental data.

\section{REFERENCES}

ANBAR, S., \& ANBAR, D. (1982). Visual masking: A unified approach. Perception, 11, 427-439.

BRAFF, D. L., \& SACCuzzo, D. P. (1981). Information processing dysfunction in paranoid schizophrenia: A two-factor deficit. American Journal of Psychiatry, 138, 1051-1056.

BREITMEYER, B. G., \& OGMEN, H. (2000). Recent models and findings in visual backward masking: A comparison, review, and update. Perception \& Psychophysics, 62, 1572-1595.

BRIDGEMAN, B. (1971). Metacontrast and lateral inhibition. Psychological Review, 78, 528-539.

BRIDGEMAN, B. (1978). Distributed sensory coding applied to simulations of iconic storage and metacontrast. Bulletin of Mathematical Biology, 40, 605-623.

Di Lollo, V., Enns, J. T., \& Rensink, R. A. (2000). Competition for consciousness among visual events: The psychophysics of reentrant visual processes. Journal of Experimental Psychology: General, 129 481-507.

EnNS, J. T., \& Di Lollo, V. (2000). What's new in visual masking? Trends in Cognitive Sciences, 4, 345-352.

FrancIs, G. (1997). Cortical dynamics of lateral inhibition: Metacontrast masking. Psychological Review, 104, 572-594.

FrANCIS, G. (2000). Quantitative theories of metacontrast masking. Psychological Review, 107, 768-785.

FRANCIS, G. (2003). Developing a new quantitative account of backward masking. Cognitive Psychology, 46, 198-226.

Francis, G., \& Herme NS, F. (2002). Comment on "Competition for consciousness among visual events: The psychophysics of reentrant visual processes" (Di Lollo, Enns, and Rensink, 2000). Journal of Experimental Psychology: General, 131, 590-593.

Green, M. F., Nuechterlein, K. H., \& Mintz, J. (1994). Backward masking in schizophrenia and mania: I. Specifying a mechanism. Archives of General Psychiatry, 51, 939-944.

Purushothaman, G., Ogmen, H., \& Bedell, H. E. (2000). Gammarange oscillations in backward-masking functions and their putative neural correlates. Psychological Review, 107, 556-577.

Slaghuis, W. L., \& Curran, C. E. (1999). Spatial frequency masking in positive- and negative-symptom schizophrenia. Journal of Abnormal Psychology, 108, 42-50.

SPERLING, G. (1960). The information available in brief visual presentations. Psychological Monographs, 74(11, Whole No. 498).

WEISSTEIN, N. (1968). A Rashevsky-Landahl neural net: Simulation of metacontrast. Psychological Review, 75, 494-521.

Weisstein, N. (1972). Metacontrast. In D. Jameson \& L. Hurvich (Eds.), Handbook of sensory physiology: Vol. 7, Pt. 4, Visual psychophysics (pp. 233-272). Berlin: Springer-Verlag.

(Manuscript received July 12, 2002; revision accepted for publication March 29, 2003. ) 